PROCEEDINGS OF THE

AMERICAN MATHEMATICAL SOCIETY

Volume 128, Number 6, Pages 1647-1653

S 0002-9939(99)05569-0

Article electronically published on November 24, 1999

\title{
HILBERT NORMS FOR GRADED ALGEBRAS
}

\author{
JOACHIM KUPSCH AND OLEG G. SMOLYANOV
}

(Communicated by Dale Alspach)

\begin{abstract}
This paper presents a solution to a problem from superanalysis about the existence of Hilbert-Banach superalgebras. Two main results are derived:

1) There exist Hilbert norms on some graded algebras (infinite-dimensional superalgebras included) with respect to which the multiplication is continuous.

2) Such norms cannot be chosen to be submultiplicative and equal to one on the unit of the algebra.
\end{abstract}

\section{InTRODUCTION}

The type of norms investigated in this article are generalizations of norms used for the symmetric tensor algebra in the white noise analysis [HKPS93, KK93. or in the Malliavin calculus Wat84. But now more general algebras are included, especially the algebra of antisymmetric tensors (Grassmann algebra) and $\mathbf{Z}_{2}$-graded algebras (superalgebras) related to supersymmetry and to quantum probability Mey93.

A locally convex commutative superalgebra is a $\mathbf{Z}_{2}$-graded locally convex space $\mathcal{E}=\mathcal{E}_{0} \oplus \mathcal{E}_{1}$ equipped with an associative continuous multiplication having the following property: for any $a, b \in \mathcal{E}_{0} \cup \mathcal{E}_{1}, a b \neq 0$ the product satisfies $a b=$ $(-1)^{p(a) p(b)} b a$ with the parity function $p$, which is defined on $\left(\mathcal{E}_{0} \cup \mathcal{E}_{1}\right) \backslash\{0\}$ with $p\left(\mathcal{E}_{0} \backslash\{0\}\right)=0, p\left(\mathcal{E}_{1} \backslash\{0\}\right)=1$, and $p(a b)=|p(a)-p(b)|$. Typical examples are Grassmann algebras with finite or countable sets of generators. In superanalysis one considers modules over (commutative) superalgebras [Rog80], [JP81], [DeW84], VV85, Rog86, Ber87], SS88, [Khr881 1 It is quite easy to define an infinitedimensional Grassmann algebra with a non-Hilbertian norm Rog80. But for a long time it was unknown whether the topology of a locally convex superalgebraincluding the Grassmann algebra - can be defined with a Hilbert norm, and moreover, whether this norm can be chosen to be simultaneously submultilplicative and equal to one at the unit of the algebra. The paper gives a complete solution to these problems. Our theorems imply a positive answer to the first question and a negative answer to the second question.

Received by the editors February 20, 1998 and, in revised form, August 3, 1998.

2000 Mathematics Subject Classification. Primary 16W50, 16W55; Secondary 46C05, 46H25.

The second author was supported in part by the Deutsche Forschungsgemeinschaft (DFG) and by the Russian Fund of Fundamental Research.

${ }^{1}$ In the pioneering works of Martin [Mar59] and of Berezin Ber66 the Grassmann algebra itself has been used instead of these modules. 


\section{General considerations}

Let $\mathcal{A}$ be an algebra over the field $\mathbf{K}=\mathbf{R}$ or $\mathbf{C}$ with unit $e_{0}$. The product is denoted by $a, b \in \mathcal{A} \rightarrow a b \in \mathcal{A}$. We assume that $\mathcal{A}$ is provided with a positive definite inner product $a, b \in \mathcal{A} \rightarrow(a \mid b) \in \mathbf{K}$. The corresponding Hilbert norm $\|a\|=\sqrt{(a \mid a)} \geq 0$ is normalized at the unit $\left\|e_{0}\right\|=1$. We are interested in such norms which allow a uniform estimate for the product of the algebra

$$
\|a b\| \leq \gamma\|a\|\|b\|
$$

with a constant $\gamma \geq 1$. In this section we prove under rather general conditions that this constant has the lower limit $\gamma \geq \sqrt{\frac{4}{3}}$.

Theorem 1. Let $\mathcal{A}$ be an algebra over the field $\mathbf{K}=\mathbf{R}$ or $\mathbf{C}$ with dimension $\operatorname{dim} \mathcal{A} \geq 2$. If this algebra satisfies the properties

i) $\mathcal{A}$ is provided with a Hilbert inner product (.|.) normalized at the unit $e_{0}$, $\left\|e_{0}\right\|^{2}=\left(e_{0} \mid e_{0}\right)=1$,

ii) there exists at least one element $f \in \mathcal{A}, f \neq 0$, such that $e_{0}, f$ and $f^{2}=f f$ satisfy $\left(e_{0} \mid f\right)=\left(f \mid f^{2}\right)=0$ and $\left(e_{0} \mid f^{2}\right) \geq 0$,

then the norm estimate $\|a b\| \leq \gamma\|a\|\|b\|$ is not valid for some $a, b \in \mathcal{A}$, if $\gamma<\sqrt{\frac{4}{3}}$.

Proof. Since $f \neq 0$, we can normalize this element and assume $\|f\|=1$. Take $a=e_{0}+\lambda f$ with $\lambda \in \mathbf{R}$. Then $a^{2}=e_{0}+2 \lambda f+\lambda^{2} f^{2}$ and $\left\|a^{2}\right\|^{2}=1+2 \lambda^{2}\left(e_{0} \mid f^{2}\right)+$ $4 \lambda^{2}+\lambda^{4}\left\|f^{2}\right\|^{2} \geq 1+4 \lambda^{2}$. On the other hand $\|a\|^{2}=1+\lambda^{2}$, and $\left\|a^{2}\right\|^{2} \leq \gamma^{2}\|a\|^{4}$ implies $1+4 \lambda^{2} \leq \gamma^{2}\left(1+\lambda^{2}\right)^{2}$. But this inequality is true for all $\lambda \geq 0$ only if $\gamma^{2} \geq \sup _{\lambda \geq 0}\left(1+4 \lambda^{2}\right)\left(1+\lambda^{2}\right)^{-2}=\frac{4}{3}$.

This theorem obviously applies to the tensor algebra $\mathcal{T}=\bigoplus_{n=0}^{\infty} \mathcal{T}_{n}$, where $\mathcal{T}_{n}$ is the subspace of tensors of degree $n$, and the norm is defined in the standard way as

$$
\|f\|^{2}=\sum_{n=0}^{\infty} w_{n}\left\|f_{n}\right\|_{n}^{2} \text { if } f=\sum_{n=0}^{\infty} f_{n}, f_{n} \in \mathcal{T}_{n},
$$

with arbitrary positive weights $w_{n}>0, n \in \mathbf{N}$ and $w_{0}=1$. In that case we can simply choose an element $f \in \mathcal{T}_{1}, f \neq 0$, to satisfy the assumptions with $\left(e_{0} \mid f \otimes f\right)=0$.

Theorem 1 can also be applied to a large class of algebras $\mathcal{A}$ which can be derived from the tensor algebra $\mathcal{T}$ by the following modifications of the product.

1. The product is generated by $f, g \in \mathcal{A}_{1}=\mathcal{T}_{1} \rightarrow f \circ g:=f \otimes g+(-1)^{\chi} g \otimes f$ where $\chi=0,1 \bmod 2$ is a parity factor.

2. The product is generated by $f, g \in \mathcal{A}_{1}=\mathcal{T}_{1} \rightarrow f \circ g:=f \otimes g+(-1)^{\chi} g \otimes$ $f+\omega(f, g) e_{0}$. Here $\chi$ is again a parity factor and $\omega(.,):. \mathcal{A}_{1} \times \mathcal{A}_{1} \rightarrow \mathbf{K}$ is a bilinear pairing.

The first class of algebras includes the algebra of symmetric tensors, the algebra of antisymmetric tensors (Grassmann algebra), and tensor products of these algebras, including the $\mathbf{Z}_{2}$-graded algebras (superalgebras) used in quantum field theory. The assumptions of Theorem 1 are satisfied for any non-vanishing element $f \in \mathcal{A}_{1}=\mathcal{T}_{1}$.

The second class includes the Clifford product, the (symmetric) Wiener product, the antisymmetric Wiener product (with antisymmetric $\omega$ ) and Le Jan's supersymmetric Wiener-Grassmann product [Jan88], Kup90], Mey93. In these cases the 
assumptions of Theorem 1 are satisfied if there exists a non-vanishing $f \in \mathcal{A}_{1}$ with $\omega(f, f) \geq 0$. Such a vector can always be found

if the algebra is complex, or

if the algebra is real and $\omega$ is not negative definite.

The last constraint is satisfied for the symmetric Wiener product on real spaces, and for the real Clifford system in quantum field theory [BSZ92. In both cases the form $\omega$ is positive definite.

Moreover Theorem 1 is obviously true for any unital algebra $\mathcal{A}$, which has a nilpotent element $f$ that is orthogonal to the unit element. If we only know that $\mathcal{A}$ has at least one nilpotent element, we can derive the weaker

Corollary 1. Let $\mathcal{A}$ be an algebra which satisfies condition i) of Theorem 11. If this algebra has a nilpotent element $f$, then the norm estimate $\|a b\| \leq\|a\|\|b\|$ is not valid for some $a, b \in \mathcal{A}$.

Proof. We assume again $\|f\|=1$. Then $a=e_{0}+\lambda f$ with $\lambda \in \mathbf{R}$ and $a^{2}=$ $\left(e_{0}+\lambda f\right)^{2}=e_{0}+2 \lambda f$ have the norms $\|a\|^{2}=1+2 \lambda \operatorname{Re}\left(e_{0}, f\right)+\lambda^{2}$ and $\left\|a^{2}\right\|^{2}=$ $1+4 \lambda \operatorname{Re}\left(e_{0}, f\right)+4 \lambda^{2}$. If $\operatorname{Re}\left(e_{0}, f\right)=0$, we can apply the arguments given in the proof for Theorem 1. If $\operatorname{Re}\left(e_{0}, f\right)=\gamma \neq 0$, then we choose $\lambda=-2 \gamma$, and $\left\|a^{2}\right\|^{2}=1+8 \gamma^{2} \leq 1=\|a\|^{4}$ is a contradiction.

\section{Norm estimates for Z-Graded Algebras}

In this section we present Hilbert norm estimates for rather general $\mathbf{Z}$-graded algebras $\mathcal{A}$ over the field $\mathbf{K}=\mathbf{R}$ or $\mathbf{C}$.. We assume the following structure of $\mathcal{A}$.

1. The algebra is the direct sum $\mathcal{A}=\bigoplus_{n=0}^{\infty} \mathcal{A}_{n}$ of orthogonal spaces $\mathcal{A}_{n}$. Thereby $\mathcal{A}_{0}$ is the one dimensional space $\mathbf{K}$ spanned by the unit $e_{0}$ of the algebra. The product $a \circ b$ maps $\mathcal{A}_{p} \times \mathcal{A}_{q}$ into $\mathcal{A}_{p+q}$ for all $p, q \in\{0,1, \ldots\}$.

2. The spaces $\mathcal{A}_{n}$ are provided with Hilbert norms $\|.\|_{n}, n=0,1, \ldots$. The unit has norm $\left\|e_{0}\right\|_{0}=1$. The product of two homogeneous elements $a_{p} \in \mathcal{A}_{p}$ and $b_{q} \in \mathcal{A}_{q}$ satisfies

$$
\left\|a_{p} \circ b_{q}\right\|_{p+q} \leq\left\|a_{p}\right\|_{p}\left\|b_{q}\right\|_{q}
$$

if $a_{p} \in \mathcal{A}_{p}$ and $b_{q} \in \mathcal{A}_{q}$.

3. The algebra is provided with a family of Hilbert norms

$$
\|a\|_{(\sigma)}^{2}=\sum_{n=0}^{\infty} w_{n}(\sigma)\left\|a_{n}\right\|_{n}^{2} \text { if } a=\sum_{n=0}^{\infty} a_{n}, a_{n} \in \mathcal{A}_{n}
$$

with $\sigma \in \mathbf{R}$. The factors $w_{n}(\sigma), n=0,1, \ldots$, are positive weights with the normalization $w_{0}(\sigma)=1$ for all $\sigma \in \mathbf{R}$. The weights satisfy the inequalities $w_{n}(\sigma) \leq w_{n}(\tau)$ for all $n \in \mathbf{N}$ if $\sigma \leq \tau$.

An immediate consequence of these assumptions is $\|a\|_{(\sigma)} \leq\|a\|_{(\tau)}$ for all $a \in \mathcal{A}$ if $\sigma \leq \tau$. A simple example of such an algebra $\mathcal{A}$ is the tensor algebra $\mathcal{T}$. Its standard norm satisfies (3) with weights $w_{n}=1$ for all $n=0,1, \ldots$. More interesting examples are the algebras of symmetric tensors or of antisymmetric tensors. With the notation $f \circ g$ for both the symmetric and the antisymmetric tensor product the estimate (3) is satisfied by the norms

$$
\left\|f_{1} \circ f_{2} \circ \ldots \circ f_{n}\right\|_{n}^{2}=\left\{\begin{array}{c}
(n !)^{-1} \operatorname{per}\left(f_{\mu} \mid f_{\nu}\right) \text { for symmetric tensors, } \\
(n !)^{-1} \operatorname{det}\left(f_{\mu} \mid f_{\nu}\right) \text { for antisymmetric tensors, }
\end{array}\right.
$$


but it is violated if the factor $(n !)^{-1}$ is omitted. The standard norm 2 is defined without the factor $(n !)^{-1}$. In the notations used here it corresponds therefore to a norm (4) with a weight function $w_{n}=n$ !.

Theorem 2. If there exists a constant $\delta(\sigma, \tau, \rho)>0$ such that the weight functions satisfy the inequalities

$$
(p+q-1) w_{p+q}(\rho) \leq \delta(\sigma, \tau ; \rho) w_{p}(\sigma) w_{q}(\tau) \text { if } p, q \geq 1
$$

for values of $\sigma, \tau$ and $\rho$ with $\sigma \leq \rho$ and $\tau \leq \rho$, then the product of $\mathcal{A}$ is estimated by

$$
\|a \circ b\|_{(\rho)} \leq \gamma \cdot\|a\|_{(\sigma)}\|b\|_{(\tau)}
$$

where the constant $\gamma$ is $\gamma=\sqrt{3} \max (1, \delta(\sigma, \tau, \rho))$.

Proof. For $a=a_{0}+a_{+}$and $b=b_{0}+b_{+}$with $a_{0}, b_{0} \in \mathcal{A}_{0}=\mathbf{K}$ and $a_{+}=\sum_{n=1}^{\infty} a_{n}$, $b_{+}=\sum_{n=1}^{\infty} b_{n}$ with $a_{n}, b_{n} \in \mathcal{A}_{n}, n \in \mathbf{N}$, the norm of $a \circ b$ is calculated by

$$
\begin{aligned}
& \|a \circ b\|_{(\rho)}^{2}=\left\|a_{0} b_{0}+a_{0} b_{+}+a_{+} b_{0}+a_{+} \circ b_{+}\right\|_{(\rho)}^{2} \\
& \leq\left|a_{0} b_{0}\right|^{2}+3\left(\left|a_{0}\right|^{2}\left\|b_{+}\right\|_{(\rho)}^{2}+\left\|a_{+}\right\|_{(\rho)}^{2}\left|b_{0}\right|^{2}+\left\|a_{+} \circ b_{+}\right\|_{(\rho)}^{2}\right) \\
& \leq\left|a_{0} b_{0}\right|^{2}+3\left(\left|a_{0}\right|^{2}\left\|b_{+}\right\|_{(\rho)}^{2}+\left\|a_{+}\right\|_{(\rho)}^{2}\left|b_{0}\right|^{2}+\sum_{n \geq 1} w_{n}(\rho)\left\|\sum_{p+q=n}^{\prime} a_{p} \circ b_{q}\right\|_{n}^{2}\right) .
\end{aligned}
$$

The symbol $\sum^{\prime}$ means summation with the constraint $p \geq 1, q \geq 1$. The sum $\sum_{p+q=n, p \geq 1, q \geq 1} \ldots=\sum_{p+q=n}^{\prime} \ldots$ has $n-1$ terms; hence

$$
\left\|\sum_{p+q=n}^{\prime} a_{p} \circ b_{q}\right\|_{n}^{2} \leq(n-1) \sum_{p+q=n}^{\prime}\left\|a_{p} \circ b_{q}\right\|_{n}^{2} \stackrel{(3)}{\leq}(n-1) \sum_{p+q=n}^{\prime}\left\|a_{p}\right\|_{p}^{2}\left\|b_{q}\right\|_{q}^{2} .
$$

If $w_{n}(\rho)$ is chosen such that (6) is satisfied, we obtain

$$
\begin{aligned}
& \sum_{n \geq 1} w_{n}(\rho)\left\|\sum_{p+q=n}^{\prime} a_{p} \circ b_{q}\right\|_{n}^{2} \\
& \quad \leq \delta \cdot\left(\sum_{p \geq 1} w_{p}(\sigma)\left\|a_{p}\right\|_{p}^{2}\right) \cdot\left(\sum_{q \geq 1} w_{q}(\tau)\left\|b_{q}\right\|_{q}^{2}\right) \leq \delta\left\|a_{+}\right\|_{(\sigma)}^{2}\left\|b_{+}\right\|_{(\tau)}^{2} .
\end{aligned}
$$

For $\rho \leq \sigma, \tau$ we have in addition the inequalities $\left\|a_{+}\right\|_{(\rho)}^{2} \leq\left\|a_{+}\right\|_{(\sigma)}^{2}$ and $\left\|b_{+}\right\|_{(\rho)}^{2} \leq$ $\left\|b_{+}\right\|_{(\tau)}^{2}$ such that finally

$$
\begin{aligned}
\|a \circ b\|_{(\rho)}^{2} & \leq\left|a_{0} b_{0}\right|^{2}+3\left(\left|a_{0}\right|^{2}\left\|b_{+}\right\|_{(\tau)}^{2}+\left\|a_{+}\right\|_{(\sigma)}^{2}\left|b_{0}\right|^{2}+\delta\left\|a_{+}\right\|_{(\sigma)}^{2}\left\|b_{+}\right\|_{(\tau)}^{2}\right) \\
& \leq 3 \gamma\|a\|_{(\sigma)}^{2}\|b\|_{(\tau)}^{2},
\end{aligned}
$$

where $\gamma$ is $\gamma=\max (1, \delta)$.

\footnotetext{
${ }^{2}$ The "standard" inner product of the symmetric/antisymmetric tensor algebra is characterized by the following property. Let $\mathcal{F}_{i}, i=1,2$, be two orthogonal subspaces of the space $\mathcal{A}_{1}$. Denote by $\mathcal{A}\left(\mathcal{F}_{i}\right)$ the subalgebra generated by elements $f \in \mathcal{F}_{i}$. Then $\left(a_{1} \circ a_{2} \mid b_{1} \circ b_{2}\right)=\left(a_{1} \mid b_{1}\right)\left(a_{2} \mid b_{2}\right)$ holds for all $a_{i} \in \mathcal{A}\left(\mathcal{F}_{i}\right), i=1,2$.
} 
As the first application of Theorem [2] we derive norms with respect to which the product of the algebra is continuous. In that case the inequality (6) has to be satisfied for identical weights $w_{p}(\sigma)=w_{p}(\tau)=w_{p}(\rho)=w_{p}, p \geq 1$. If we fix $q=1$, then (6) implies $p \cdot w_{p+1} \leq \delta \cdot w_{p} \cdot w_{1}$ for $p \in \mathbf{N}$. As a consequence we obtain $w_{p} \leq \delta^{p-1}((p-1) !)^{-1} w_{1}, p \geq 1$. The slowest decrease of the weights which might be possible according to our estimates is therefore $w_{p} \sim((p-1) !)^{-1}$. The proof that such a solution actually exists follows from the simple estimate $(m+n) ! \geq m ! n !$ if $m, n \geq 0$. Hence $(p+q-1) \frac{1}{(p+q-1) !}=\frac{1}{(p+q-2) !} \leq \frac{1}{(p-1) !} \frac{1}{(q-1) !}$ is valid for all $p, q \geq 1$. Since

$$
2^{m+n} \geq\left(\begin{array}{c}
m+n \\
m
\end{array}\right)=\frac{(m+n) !}{m ! n !} \geq m+n \text { if } m, n \geq 1,
$$

also $(p+q-1) \frac{1}{(p+q) !}<\frac{1}{(p+q-1) !} \leq \frac{1}{p !} \frac{1}{q !}$ follows for all $p, q \geq 1$. We have therefore derived

Corollary 2. If the norm is defined with the weights $w_{0}=1, w_{n}=((n-1) !)^{-1}$, $n \geq 1$, or with $w_{0}=1, w_{n}=(n !)^{-1}, n \geq 1$, the product of the algebra is continuous with the uniform norm estimate

$$
\|a \circ b\| \leq \sqrt{3}\|a\|\|b\| .
$$

As a more general class of norms we choose weights

$$
w_{0}=1, w_{n}(\sigma, \rho, s)=(n !)^{\sigma} 2^{\rho n}(1+n)^{s} \text { if } n \geq 1,
$$

with real parameters $\sigma, \rho, s$. These weights satisfy the inequalities $w_{n}\left(\sigma_{1}, \rho_{1}, s_{1}\right) \leq$ $w_{n}\left(\sigma_{2}, \rho_{2}, s_{2}\right)$ if $\sigma_{1} \leq \sigma_{2}, \rho_{1} \leq \rho_{2}, s_{1} \leq s_{2}$. We denote by $\|a\|_{(\sigma, \rho, s)}$ the norm (4) defined with the weights $w_{n}(\sigma, \rho, s)$. The estimate (8) and the bounds $\frac{(m+n) !}{m ! n !} \geq$ $\frac{(2 m) !}{(m !)^{2}} \geq$ const $\cdot 2^{2 m} m^{-\frac{1}{2}}$ if $n \geq m \geq 1$ and $1 \leq \frac{(1+m)(1+n)}{1+m+n} \leq 1+\min (m, n)$ yield inequalities of the type (6) also for these norms. We obtain

$$
(p+q-1) w_{p+q}(\sigma, \rho, s) \leq \delta w_{p}\left(\sigma^{\prime}, \rho^{\prime}, s^{\prime}\right) w_{q}\left(\sigma^{\prime}, \rho^{\prime}, s^{\prime}\right) \text { if } p, q \geq 1
$$

with a constant $\delta \geq 1$ if $\sigma=\sigma^{\prime}=-1$ with $\rho=\rho^{\prime} \in \mathbf{R}$ and $s=s^{\prime} \leq 0$, or if $\sigma=\sigma^{\prime}<-1$ with $\rho=\rho^{\prime} \in \mathbf{R}$ and $s=s^{\prime} \in \mathbf{R}$.

The generalizations of (92) are therefore

$$
\|a \circ b\|_{(-1, \rho, s)} \leq \sqrt{3}\|a\|_{(-1, \rho, s)} \cdot\|b\|_{(-1, \rho, s)} \text { if } \rho \in \mathbf{R}, s \leq 0
$$

and

$$
\|a \circ b\|_{(\sigma, \rho, s)} \leq \gamma\|a\|_{(\sigma, \rho, s)} \cdot\|b\|_{(\sigma, \rho, s)} \text { if } \sigma<-1, \rho \in \mathbf{R}, s \in \mathbf{R} .
$$

Here $\gamma$ takes some value $\gamma \geq \sqrt{3}$ depending on the choice of the parameters $\sigma$ and $s$.

Moreover, the inequalities (11) are valid for $(\sigma, \rho, s) \neq\left(\sigma^{\prime}, \rho^{\prime}, s^{\prime}\right)$ if $\sigma<\sigma^{\prime}$ or if $\sigma=\sigma^{\prime}$ and $\rho<\rho^{\prime}$. The corresponding estimates for the norms are

$$
\|a \circ b\|_{(\sigma, \rho, s)} \leq \gamma\|a\|_{\left(\sigma^{\prime}, \rho^{\prime}, s^{\prime}\right)} \cdot\|b\|_{\left(\sigma^{\prime}, \rho^{\prime}, s^{\prime}\right)} \text { if } \sigma<\sigma^{\prime} \text { for all } \rho, \rho^{\prime}, s, s^{\prime} \in \mathbf{R},
$$

and

$$
\|a \circ b\|_{(\sigma, \rho, s)} \leq \gamma\|a\|_{\left(\sigma, \rho^{\prime}, s^{\prime}\right)} \cdot\|b\|_{\left(\sigma, \rho^{\prime}, s^{\prime}\right)} \text { if } \rho<\rho^{\prime} \text { for all } \sigma, s, s^{\prime} \in \mathbf{R} .
$$

The value of $\gamma \geq \sqrt{3}$ depends on the choice of the parameters. 
For the tensor algebra and for algebras of symmetrized tensor: 3 the Hilbert space $\mathcal{A}_{1}=\mathcal{H}$ generates the whole algebra. Given a (self-adjoint/positive) operator $A$ on $\mathcal{H}$, the mapping $\Gamma(A) e_{0}=e_{0}$ and $\Gamma(A)\left(f_{1} \circ f_{2} \circ \ldots \circ f_{n}\right):=\left(A f_{1}\right) \circ\left(A f_{2}\right) \circ \ldots \circ\left(A f_{n}\right)$ for $f_{\mu} \in \mathcal{H}, \mu=1, \ldots, n$, and $n \in \mathbf{N}$, defines a unique (self-adjoint/positive) operator $\Gamma(A)$ on the algebra $\mathcal{A}$, which satisfies the relation

$$
\Gamma(A)(a \circ b)=(\Gamma(A) a) \circ(\Gamma(A) b) .
$$

The norms (4) with the weights (10) are then easily generalized to

$$
\|a\|_{(\sigma, \rho, s)}^{2}=\sum_{n=0}^{\infty}(n !)^{\sigma}\left\|(\Gamma(A))^{\rho} a_{n}\right\|_{n}^{2}(1+n)^{s} \text { if } a=\sum_{n=0}^{\infty} a_{n}, a_{n} \in \mathcal{A}_{n} .
$$

If $A$ is an invertible positive operator with lower bound $A \geq 2 \cdot i d$, then $\Gamma(A)$ satisfies $\left\|(\Gamma(A))^{-\rho} a\right\|_{n} \leq 2^{-n \rho}\|a\|_{n}$ for $a \in \mathcal{A}_{n}$ if $\rho \geq 0$. This bound and the relation (16) imply that the estimates (12), (13) and (15) are also valid for the norms (17); moreover (14) holds if $\rho \leq \rho^{\prime}$.

If $A^{-1}$ is a Hilbert-Schmidt operator, then a family of norms (17) can be used to define a nuclear topology on the algebra $\mathcal{A}$. For the symmetric tensor algebra that has been done in the white noise calculus and in the Malliavin calculus, see e.g. [AM93], [KS93, Wat84]. For the algebra of antisymmetric tensors and for the superalgebras such nuclear topologies can be found in [Kré78 and in [HK95]. But the estimates of these references are not strong enough to derive the results with a single Hilbert norm as presented in Corollary 2 and in equations (12) and (13).

\section{REFERENCES}

[AM93] A. Arai and I. Mitoma, Comparison and nuclearity of spaces of differential forms on topological vector spaces, J. Funct. Anal. 111 (1993), 278-294. [MR 94a:46051]

[Ber66] F. A. Berezin, The method of second quantization, Academic Press, New York, 1966. MR 34:8738

[Ber87] F. A. Berezin, Introduction to Superanalysis, Reidel, Dordrecht, 1987. MR 89b:58006

[BSZ92] J. C. Baez, I. E. Segal, and Z. Zhou, Introduction to Algebraic and Constructive Quantum Field Theory, Princeton University Press, Princeton, 1992. MR 93m:81002

[DeW84] B. DeWitt, Supermanifolds, CUP, Cambridge, 1984. MR 87b:58007

[HK95] Z. Haba and J. Kupsch, Supersymmetry in euclidean quantum field theory, Fortschr. Phys. 43 (1995), 41-66. MR 96d:81109

[HKPS93] T. Hida, H.-H. Kuo, J. Potthoff, and L. Streit, White noise, Kluwer, Dordrecht, 1993. MR 95f:60046

[Jan88] Y. Le Jan, On the Fock space representation of functionals of the occupation number field and their renormalization, J. Funct. Anal. 80 (1988), 88-108. [MR 89k:60100.

[JP81] A. Jadczyk and K. Pilch, Superspaces and supersymmetries, Commun. Math. Phys. 78 (1981), 373-390. MR 82e:58002

[Khr88] A. Yu. Khrennikov, Functional superanalysis, Russian Math. Surveys 43 (1988), 103137. MR 90f:58019a

[Kré78] P. Krée, Méthodes fonctionelles en analyse de dimension infinie et holomorphie anticommutative, Séminaire P. Lelong et H. Skoda (Analyse) Année 1976/77, Lect. Notes Math. 694, Springer, Berlin, 1978, pp. 134-171. MR 80m:81045

[KS93] Yu G. Kondratev and L. Streit, Spaces of white noise distributions: constructions, descriptions, applications. I, Rep. Math. Phys. 33 (1993), 341-366. MR 95m:60095]

[Kup90] J. Kupsch, A probabilistic formulation of bosonic and fermionic integration, Rev. Math. Phys. 2 (1990), 457-477. MR 93a:81100

\footnotetext{
${ }^{3}$ This class of algebras does not only include the algebra of symmetric tensors and the algebra of antisymmetric tensors (Grassmann algebra), but also the $\mathbf{Z}_{2}$-graded algebras (superalgebras) used in supersymmetric quantum field theory.
} 
[Mar59] J. L. Martin, Generalized classical dynamics, and the "classical analogue" of a Fermi oscillator, Proc. Roy. Soc. (London) A251 (1959), 536-542. MR 22:525

[Mey93] P. A. Meyer, Quantum probability for probabilists, Lect. Notes in Math. 1538, Springer, Berlin, 1993. MR 94k:81152

[Rog80] A. Rogers, A global theory of supermanifolds, J. Math. Phys. 21 (1980), 1352-1365. MR 82d:58001

[Rog86] A. Rogers, Graded manifolds, supermanifolds and infinite-dimensional Grassmann algebras, Commun. Math. Phys. 105 (1986), 375-384. MR 87f:58009

[SS88] O. G. Smolyanov and E. T. Shavgulidze, The Fourier transform and pseudodifferential operators in superanalysis, Soviet Math. Dokl. 37 (1988), 476-481. MR 89h:58030

[VV85] V. S. Vladimirov and I. V. Volovich, On the definition of the integral in superspace, Soviet Math. Dokl. 32 (1985), 817-819. MR 87g:58017

[Wat84] S. Watanabe, Lectures on Stochastic Differential Equations and Malliavin Calculus, Tata Institute, Bombay, and Springer-Verlag, Berlin, 1984. MR 86b:60113

Fachbereich Physik der Universität Kaiserslautern, D-67663 Kaiserslautern, GerMANY

E-mail address: kupsch@physik.uni-kl.de

Faculty of Mechanics and Mathematics, Moscow State University, 119899 Moscow, RUSSIA

E-mail address: smolian@nw.math.msu.su 